\title{
Analysis of Illumina Microbial Assemblies
}

Alicia Clum ${ }^{1}$ (aclum@lbl.gov), Brian Foster ${ }^{1}$, Jeff Froula ${ }^{1}$, Kurt LaButti $^{1}$, Alex Sczyrba ${ }^{1}$, Alla Lapidus ${ }^{1}$ and Tanja Woyke ${ }^{1}$ Lawrence Berkeley National Laboratories

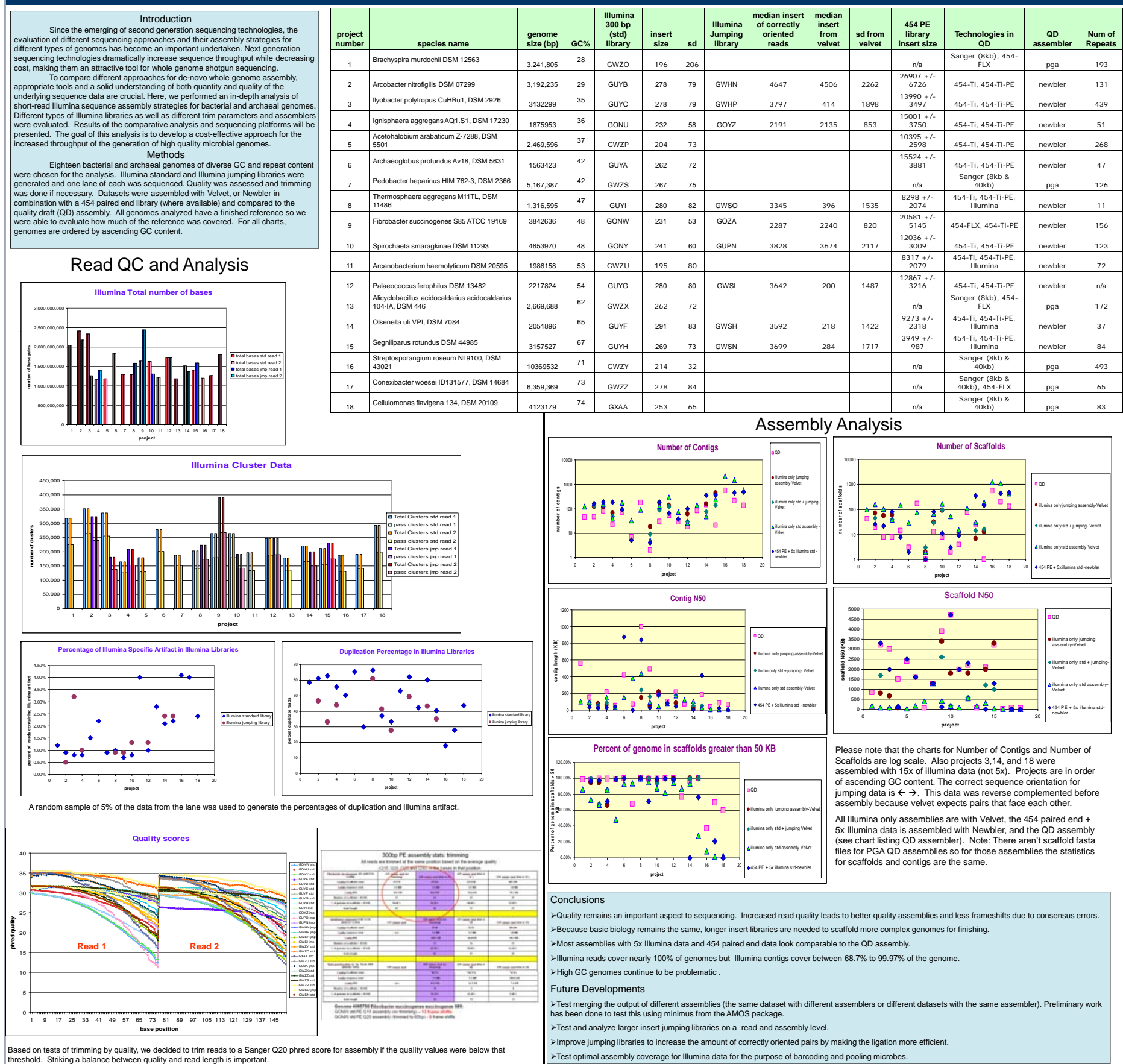

Alignment Analysis Read Coverage

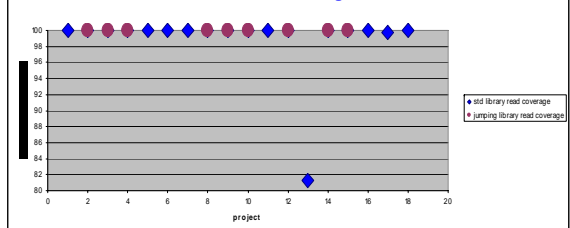

In most cases Illumina reads cover $1000 \%$ of the genome. Assembled contigs,
howevere, cover between $68.7 \%$, $1099.97 \%$ of the genomeme.
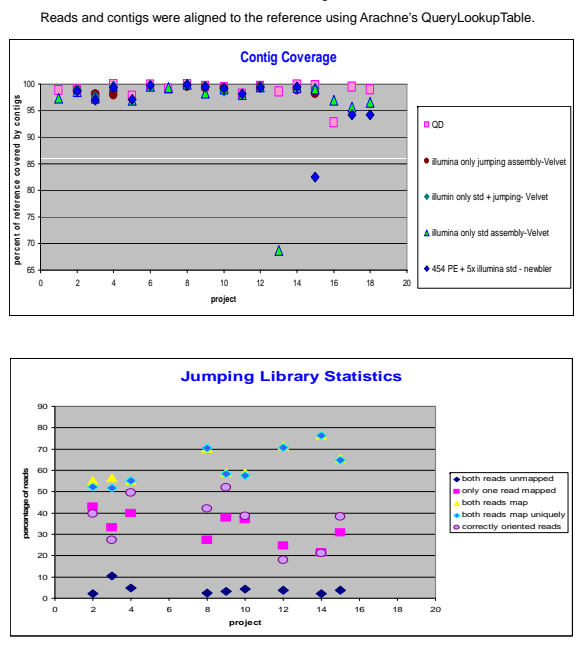

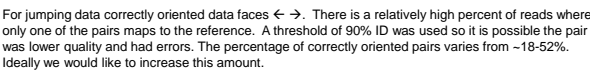

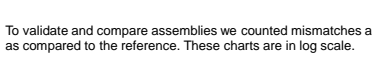
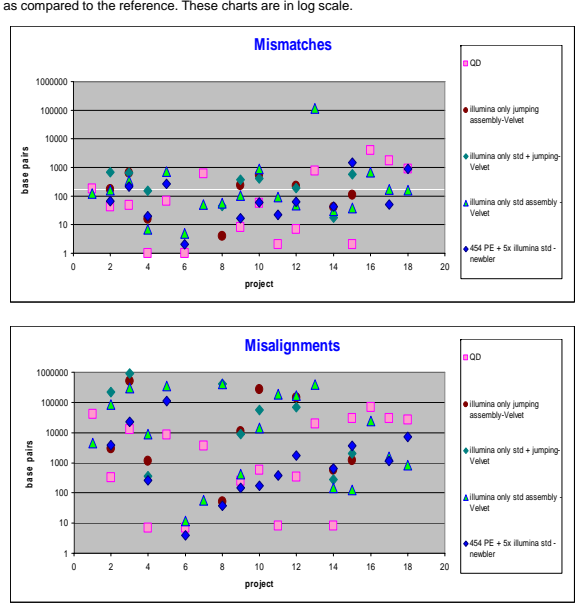